\title{
Long-Term Nitrate Therapy After Acute Myocardial Infarction Does not Improve or Aggravate Prognosis
}

\author{
Sunao Kojima, MD; Kunihiko Matsui, MD*; Tomohiro Sakamoto, MD; \\ Masaharu Ishihara, MD**; Kazuo Kimura, MD ${ }^{\dagger}$; Shunichi Miyazaki, MD ${ }^{\dagger \dagger}$; \\ Masakazu Yamagishi, MD ${ }^{\dagger}$; Chuwa Tei, MD¥; Hisatoyo Hiraoka, MD\$; \\ Masahiro Sonoda, MD\$; Kazufumi Tsuchihashi, MD\$; \\ Nobuo Shimoyama, MDI; Takashi Honda, MDII; \\ Yasuhiro Ogata, MD***; Hisao Ogawa; MD on behalf \\ of the Japanese Acute Coronary Syndrome Study (JACSS) Investigators
}

\begin{abstract}
Background There is conflicting information about whether nitrate treatment aggravates long-term prognosis, so the present retrospective study was designed to determine the effects of long-term nitrate therapy on major adverse events after acute myocardial infarction (AMI) in the coronary interventional era.

Methods and Results Using the Japanese Acute Coronary Syndrome Study database, 1,236 consecutive patients who were hospitalized within $48 \mathrm{~h}$ of onset of symptoms of AMI from January to December 2003 were evaluated. All-cause mortality, cardiac events and cardiovascular events were lower in patients treated with nitrates than in the untreated controls. However, these crude comparisons included several confounding factors on nitrate prescription. To minimize the effect of selection bias on outcomes, the technique of propensity score matching for clinical characteristics was used and distortion of effective nitrate treatment was excluded as much as possible. The results of propensity score matching showed that nitrate therapy had no impact on all-cause mortality, cardiac events and cardiovascular events at 30, 60 or 90 days, 6 months, 1 year, and 2 years follow-up.

Conclusions Long-term nitrate therapy after AMI neither improves nor aggravates prognosis. Prospective randomized clinical trials are warranted to determine the effects of long-term nitrate therapy for secondary prevention of AMI. (Circ J 2007; 71: 301-307)
\end{abstract}

Key Words: Myocardial infarction; Nitrates; Prognosis; Propensity score

$\mathbf{N}$ itrates are commonly used in patients with coronary artery disease because they relax vascular smooth muscle and the vasodilator effects are evident in systemic arteries including coronary vessels! These effects are also evident in systemic veins and the venodilator effect

(Received October 5, 2006; revised manuscript received December 4, 2006; accepted December 15, 2006)

Department of Cardiovascular Medicine, Graduate School of Medical Sciences, Kumamoto University, *Department of General Medicine, Kumamoto University Hospital, Kumamoto, **Department of Cardiology, Hiroshima City Hospital, Hiroshima, 'Division of Cardiology, Yokohama City University Medical Center, Yokohama, Division of Cardiology, Department of Internal Medicine, National Cardiovascular Center, Suita, $\$$ Department of Cardiovascular Respiratory and Metabolic Medicine, Graduate School of Medicine, Kagoshima University, Kagoshima, Department of Internal Medicine and Molecular Science, Graduate School of Medicine, Osaka University, Suita, $\$$ Second Department of Cardiology, National Hospital Kyushu Cardiovascular Center, Kagoshima, \$\$Second Department of Internal Medicine, Sapporo Medical University School of Medicine, Sapporo, IDivision of Cardiology, Oita National Hospital, Oita, IIICardiovascular Center, Saiseikai Kumamoto Hospital and ***Department of Cardiology, Japanese Red Cross Kumamoto Hospital, Kumamoto, Japan

The Japanese Acute Coronary Syndrome Study (JACSS) Investigators and participating institutions are listed in Appendix 1.

Mailing address: Sunao Kojima, MD, Department of Cardiovascular Medicine, Graduate School of Medical Sciences, Kumamoto University, 1-1-1 Honjo, Kumamoto 860-8556, Japan. E-mail: kojimas@ kumamoto-u.ac.jp reduces ventricular preload, which in turn reduces myocardial wall stress and oxygen requirements. The reduction of preload and afterload is used in the treatment of heart failure as well as for angina pectoris? The effectiveness of short-term administration of nitrates during the acute phase of myocardial infarction (MI) is well established? In 1993, the United States Food and Drug Administration Cardiorenal Drugs Advisory Committee concluded in "The Pink Sheet" that oral anti-anginal nitrates should be indicated only for single, not chronic, use in the absence of long-term data. Two megatrials regarding nitrate therapy have been conducted so far. In Gruppo Italiano per lo studio della sopravvivenza nell'infarto miocardico (GISSI-3 trial), administration of transdermal nitroglycerin for 6 weeks after acute MI (AMI) demonstrated 6\% risk reduction of overall mortality, however, the effect was not statistically significant 5 In the Fourth International Study of Infarct Survival (ISIS-4) trial involving 58,050 patients, administration of mononitrate after AMI did not demonstrate any survival benefits in the first 5 weeks. However, in both those trials, nitrates were administered for less than 2 months and thus, the long-term effects of treatment after AMI is not clear to date. What is common to those 2 megatrials is the short period of nitrate administration and that they were carried out in the fibrinolytic era. In the coronary pre-interventional era, Ishikawa et al first reported that long-term nitrate treatment increased cardiac events in patients with healed MI? Kanamasa et $\mathrm{al}^{8}$ also showed a higher incidence of car- 
Table 1 Clinical Characteristics of the 2 Study Groups

\begin{tabular}{|c|c|c|c|}
\hline & $\begin{array}{l}\text { Nitrate group } \\
\quad(n=312)\end{array}$ & $\begin{array}{c}\text { Control group } \\
(n=924)\end{array}$ & $p$ value \\
\hline Age (years)* & $70 \pm 12$ & $68 \pm 12$ & 0.0213 \\
\hline Men & $70 \%$ & $70 \%$ & 0.8456 \\
\hline Hypertension & $67 \%$ & $60 \%$ & 0.0321 \\
\hline$D M$ & $38 \%$ & $32 \%$ & 0.0654 \\
\hline Hyperlipidemia & $37 \%$ & $35 \%$ & 0.5702 \\
\hline$B M I\left(\mathrm{~kg} / \mathrm{m}^{2}\right)$ & $24 \pm 3$ & $24 \pm 3$ & 0.8837 \\
\hline Current smoker & $39 \%$ & $47 \%$ & 0.0196 \\
\hline Serum creatinine $(m g / d l) *$ & $1.1 \pm 1.1$ & $1.0 \pm 0.9$ & 0.0092 \\
\hline Previous MI & $17 \%$ & $11 \%$ & 0.0123 \\
\hline Preinfarction angina pectoris & $40 \%$ & $35 \%$ & 0.0990 \\
\hline Time from symptom onset $(h)^{*}$ & $6.4 \pm 8.5$ & $6.2 \pm 8.0$ & 0.7415 \\
\hline ST-elevation MI & $86 \%$ & $83 \%$ & 0.3709 \\
\hline$Q$-wave infarction & $68 \%$ & $69 \%$ & 0.6956 \\
\hline Peak $C K(I U / L) *$ & $2,643 \pm 2,360$ & $2,780 \pm 2,728$ & 0.4061 \\
\hline Coronary multi-vessel involvement & $49 \%$ & $40 \%$ & 0.0097 \\
\hline \multicolumn{4}{|l|}{ Culprit location } \\
\hline$L A D$ & $40 \%$ & $44 \%$ & 0.1955 \\
\hline$L C X$ & $11 \%$ & $14 \%$ & 0.1535 \\
\hline$R C A$ & $38 \%$ & $33 \%$ & 0.0908 \\
\hline \multicolumn{4}{|l|}{ Killip class } \\
\hline II & $8 \%$ & $6 \%$ & 0.1050 \\
\hline III & $4 \%$ & $3 \%$ & 0.1197 \\
\hline IV & $4 \%$ & $7 \%$ & 0.0882 \\
\hline Emergency PCI & $71 \%$ & $82 \%$ & $<0.0001$ \\
\hline \multicolumn{4}{|c|}{ In-hospital medications and after discharge } \\
\hline Aspirin & $94 \%$ & $95 \%$ & 0.4668 \\
\hline$\beta$-blocker & $37 \%$ & $35 \%$ & 0.5209 \\
\hline Calcium-channel blocker & $33 \%$ & $26 \%$ & 0.0081 \\
\hline$A C E I$ & $47 \%$ & $48 \%$ & 0.8913 \\
\hline
\end{tabular}

*Data are mean $\pm S D$.

$D M$, diabetes mellitus; BMI, body mass index; MI, myocardial infarction; $C K$, creatine kinase; LAD, left anterior descending artery; LCX, left circumflex artery; RCA, right coronary artery; PCI, percutaneous coronary intervention; ACEI, angiotensin-converting enzyme inhibitor.

diac events in patients with severe AMI on long-term and continuous use of long-acting nitrates compared with those without nitrate administration. However, the overall randomization rate was rather low and data were not adjusted for patient background in either of these studies. Nagao et al indicated that there was no significant difference in the incidence of cardiac events within 5 years after AMI in patients on long-term nitrate administration than in those without, after adjustment for age? In Japan, emergency percutaneous coronary intervention (PCI) is currently performed in approximately $80 \%$ of patients in the acute phase of MI and successful coronary reperfusion of the culprit lesion is reported in approximately $90 \% !^{10-12}$ There is no clinical evidence that long-term nitrate treatment has any clinical benefit in patients undergoing PCI for AMI.

The present study was designed to determine the effects of long-term nitrate therapy on adverse events in patients with AMI in the coronary interventional era.

\section{Methods}

\section{Data Sources}

The Japanese Acute Coronary Syndrome Study (JACSS) is a retrospective and multicenter observational study conducted at 35 medical institutions in Japan. The JACSS database includes 1,236 consecutive patients hospitalized at the participating institutions within $48 \mathrm{~h}$ of the onset of symptoms of AMI from January to December 2003. AMI was defined as elevated myocardial enzyme concentrations, with either typical chest pain persisting longer than $30 \mathrm{~min}$ or ECG changes (including ischemic ST-segment depression, ST-segment elevation, or pathologic Q waves). Elevated enzyme concentrations were defined as peak creatine kinase (CK) levels more than twice the normal upper limit. The study protocol was reviewed and approved by the ethical committee of each participating institution.

\section{Patients and Nitrate Prescription}

After hospital admission, nitrates were administered according to the priority of the attending physician. The 1,236 patients were divided into a nitrate group consisting of 312 patients who continuously received long-acting nitrates (long-acting oral isosorbide mononitrate, long-acting oral isosorbide dinitrate, long-acting transdermal nitroglycerin, or long-acting transdermal isosorbide dinitrate) and a control group consisting of 924 patients who were not prescribed long-acting nitrates during hospitalization and never received them after discharge. The clinical data were obtained from the medical records of in- and outpatients of each hospital. Patients who were treated with antihypertensive drugs or those whose baseline blood pressure was $\geq 140 / 90 \mathrm{mmHg}$ were considered hypertensive. Diabetes mellitus was diagnosed according to the criteria of the World Health Organization ${ }^{13}$ Hyperlipidemia was defined as total cholesterol $\geq 220 \mathrm{mg} / \mathrm{dl}$ and $/$ or triglyceride $\geq 150 \mathrm{mg} / \mathrm{dl}$. Cigarette smoking was defined as active smoking.

\section{Coronary Angiography and Reperfusion Therapy}

The allocation of coronary angiography and reperfusion therapy were determined by the physician. The perfusion 
Table 2 Total Deaths, Cardiac Events and Cardiovascular Events for Nitrate and Control Groups at Different Time Intervals

\begin{tabular}{|c|c|c|c|c|c|}
\hline \multirow{2}{*}{ Duration of treatment } & \multicolumn{2}{|c|}{ Nitrate $(n=312)$} & \multicolumn{2}{|c|}{ Control $(n=924)$} & \multirow{2}{*}{$p$ value } \\
\hline & $(+)$ & $(-)$ & $(+)$ & $(-)$ & \\
\hline \multicolumn{6}{|l|}{ Total deaths } \\
\hline 30 days & $7(2.2)$ & 305 (97.8) & $55(6.0)$ & $869(94.0)$ & 0.0095 \\
\hline 60 days & $7(2.2)$ & $305(97.8)$ & $55(6.0)$ & $869(94.0)$ & 0.0095 \\
\hline 90 days & $8(2.6)$ & $304(97.4)$ & $57(6.2)$ & $867(93.8)$ & 0.0136 \\
\hline 6 months & $11(3.5)$ & $301(96.5)$ & $59(6.4)$ & $865(93.6)$ & 0.0588 \\
\hline 1 year & $12(3.8)$ & $300(96.2)$ & $59(6.4)$ & 865 (93.6) & 0.0956 \\
\hline 2 years & $13(4.2)$ & $299(95.8)$ & $62(6.7)$ & $862(93.3)$ & 0.1037 \\
\hline \multicolumn{6}{|l|}{ Cardiac events* } \\
\hline 30 days & $14(4.5)$ & $298(95.5)$ & $77(8.3)$ & $847(91.7)$ & 0.0245 \\
\hline 60 days & $16(5.4)$ & $296(94.6)$ & $79(8.6)$ & $845(91.4)$ & 0.0498 \\
\hline 90 days & $20(6.4)$ & $292(93.6)$ & $81(8.8)$ & $843(91.2)$ & 0.1890 \\
\hline 6 months & $26(8.3)$ & $286(91.7)$ & $90(9.7)$ & $834(90.3)$ & 0.4612 \\
\hline 1 year & $32(10.3)$ & $280(89.7)$ & $92(10.0)$ & $832(90.0)$ & 0.8789 \\
\hline 2 years & $36(11.5)$ & $276(88.5)$ & $98(10.6)$ & $826(89.4)$ & 0.6471 \\
\hline \multicolumn{6}{|l|}{ Cardiovascular events** } \\
\hline 30 days & $16(5.1)$ & $296(94.9)$ & $84(9.1)$ & $840(90.9)$ & 0.0265 \\
\hline 60 days & $19(6.1)$ & $293(93.9)$ & $87(9.4)$ & $837(90.6)$ & 0.0697 \\
\hline 90 days & $23(7.4)$ & $289(92.6)$ & $89(9.6)$ & $835(90.4)$ & 0.2292 \\
\hline 6 months & $30(9.6)$ & $282(90.4)$ & $98(10.6)$ & $826(89.4)$ & 0.6195 \\
\hline 1 year & $36(11.5)$ & $276(88.5)$ & $100(10.8)$ & $824(89.2)$ & 0.7268 \\
\hline 2 years & $42(13.5)$ & $270(86.5)$ & $106(11.5)$ & $818(88.5)$ & 0.3493 \\
\hline
\end{tabular}

Data are number of cases (percent).

*Cardiac death, non-fatal reinfarction, unstable angina, heart failure requiring rehospitalization.

**Cardiac death, non-fatal reinfarction, unstable angina, heart failure requiring rehospitalization, stroke.

grade of the infarct-related artery was assessed in accordance with the Thrombolysis in Myocardial Infarction (TIMI) study classification! ${ }^{4}$ The final TIMI flow grade was assessed on the final image of the emergency coronary angiography.

\section{Mortality and Cardiovascular Events}

The primary endpoint was mortality from any cause. Cardiac events (cardiac death, non-fatal reinfarction, unstable angina and heart failure requiring emergency rehospitalization) and cardiovascular events (stroke in addition to cardiac events) were also assessed. We evaluated the 30-day, 60-day, 90-day, 6-month, 1-year, and 2-year adverse event rates following AMI.

\section{Statistical Analysis}

Univariate comparisons of clinical characteristics were carried out between the nitrate group and the control group using chi-square test for dichotomous variables and unpaired t-test for continuous variables. Clinical characteristics considered to be associated with nitrate administration included age, sex, background illness (hypertension, diabetes mellitus, hyperlipidemia), smoking, serum creatinine levels, ST-elevation and Q-wave MI on electrocardiographic fingings, admission characteristics (body mass index (BMI), previous MI, preinfarction angina pectoris), time from symptom onset, coronary angiographic findings (multivessel involvement, culprit location), peak CK levels and medications during hospitalization and after discharge. Killip classes on hospital admission, depending on the clinical manifestations of cardiac failure, were also assessed (Killip I, no heart failure; Killip II, S3 and/or basal lung crepitations; Killip III, acute pulmonary edema; Killip IV, cardiac shock) 15 The incidences of all-cause mortality, cardiac events, and cardiovascular events were calculated by dividing the number of events by the number of cases followed-up for the 2 groups. We tested differences between the curves of the 2 groups for statistical significance by the log-rank analysis.

In an additional effort to balance the patient groups, we used propensity score analysis to generate a set of matched cases (patients with nitrate administration) and controls (patients without nitrate administration). The propensity score was calculated for each patient based on a logistic regression analysis of the probability of nitrate prescription using clinical characteristics. With these propensity scores, we then used a greedy matching technique to create a 1-to1 match of cases with controls as described by Parsons 16 Comparisons between the 2 matched groups based on clinical characteristics were carried out using chi-square test and unpaired t-test to confirm that the groups had been successfully matched. We calculated again the incidences of all-cause mortality, cardiac events, and cardiovascular events and compared the 2 matched groups.

All analyses were performed using the SAS software package version 9.1 (SAS, Cary, NC, USA).

\section{Results}

\section{Clinical Background}

The characteristics of the patients with and without longterm nitrate administration after AMI are listed in Table 1. Of the 1,236 patients included in the study, $981(78 \%)$ underwent emergency PCI and 911 (93\%) of them achieved successful coronary reperfusion (TIMI grade 3 flow). Patients were followed-up for a mean period of $444 \pm 206$ $( \pm$ SD, maximum 738$)$ days. Long-acting nitrates were administered in 312 patients (25\%) after AMI. A comparison between groups based on clinical features revealed that patients on nitrates were more likely to be older, hypertensive, have high serum creatinine levels, previous MI and have been treated with a calcium-channel blocker. They were less likely to be current smokers and to have undergone emergency PCI. No differences were noted between the 2 groups with regard to sex, BMI, time from symptom onset to admission, peak CK, culprit location, Killip class, 
Table 3 Clinical Characteristics of the 2 Study Groups After Propensity Score Matching

\begin{tabular}{|c|c|c|c|}
\hline & $\begin{array}{l}\text { Nitrate group } \\
\quad(n=172)\end{array}$ & $\begin{array}{l}\text { Control group } \\
(n=172)\end{array}$ & $p$ value \\
\hline Age (years)* & $68 \pm 12$ & $69 \pm 11$ & 0.5021 \\
\hline Men & $70 \%$ & $66 \%$ & 0.4172 \\
\hline Hypertension & $63 \%$ & $66 \%$ & 0.5738 \\
\hline$D M$ & $37 \%$ & $30 \%$ & 0.1711 \\
\hline Hyperlipidemia & $37 \%$ & $39 \%$ & 0.6565 \\
\hline$B M I\left(\mathrm{~kg} / \mathrm{m}^{2}\right)^{*}$ & $24 \pm 4$ & $24 \pm 3$ & 0.5691 \\
\hline Current smoker & $43 \%$ & $42 \%$ & 0.8273 \\
\hline Serum creatinine $(m g / d l) *$ & $1.0 \pm 0.8$ & $0.9 \pm 0.3$ & 0.1511 \\
\hline Previous MI & $15 \%$ & $17 \%$ & 0.5591 \\
\hline Preinfarction angina pectoris & $35 \%$ & $35 \%$ & 0.9101 \\
\hline Time from symptom onset $(h)^{*}$ & $6.0 \pm 8.2$ & $5.7 \pm 7.7$ & 0.8007 \\
\hline ST-elevation MI & $87 \%$ & $90 \%$ & 0.5011 \\
\hline$Q$-wave infarction & $72 \%$ & $69 \%$ & 0.5539 \\
\hline Peak $C K(I U / L)^{*}$ & $2,817 \pm 2,439$ & $2,509 \pm 1,962$ & 0.2076 \\
\hline Coronary multi-vessel involvement & $51 \%$ & $49 \%$ & 0.7463 \\
\hline \multicolumn{4}{|l|}{ Culprit location } \\
\hline$L A D$ & $44 \%$ & $41 \%$ & 0.5858 \\
\hline$L C X$ & $9 \%$ & $10 \%$ & 0.4825 \\
\hline$R C A$ & $41 \%$ & $43 \%$ & 0.6620 \\
\hline \multicolumn{4}{|l|}{ Killip class } \\
\hline II & $8 \%$ & $7 \%$ & 0.8355 \\
\hline III & $3 \%$ & $3 \%$ & 1.0000 \\
\hline IV & $5 \%$ & $2 \%$ & 0.2398 \\
\hline Emergency $P C I$ & $87 \%$ & $88 \%$ & 0.6248 \\
\hline \multicolumn{4}{|c|}{ In-hospital medications and after discharge } \\
\hline Aspirin & $97 \%$ & $96 \%$ & 0.7774 \\
\hline$\beta$-blocker & $38 \%$ & $41 \%$ & 0.5809 \\
\hline Calcium-channel blocker & $31 \%$ & $35 \%$ & 0.3595 \\
\hline ACEI & $48 \%$ & $50 \%$ & 0.7463 \\
\hline
\end{tabular}

*Data are mean $\pm S D$.

Abbreviations see in Table 1.

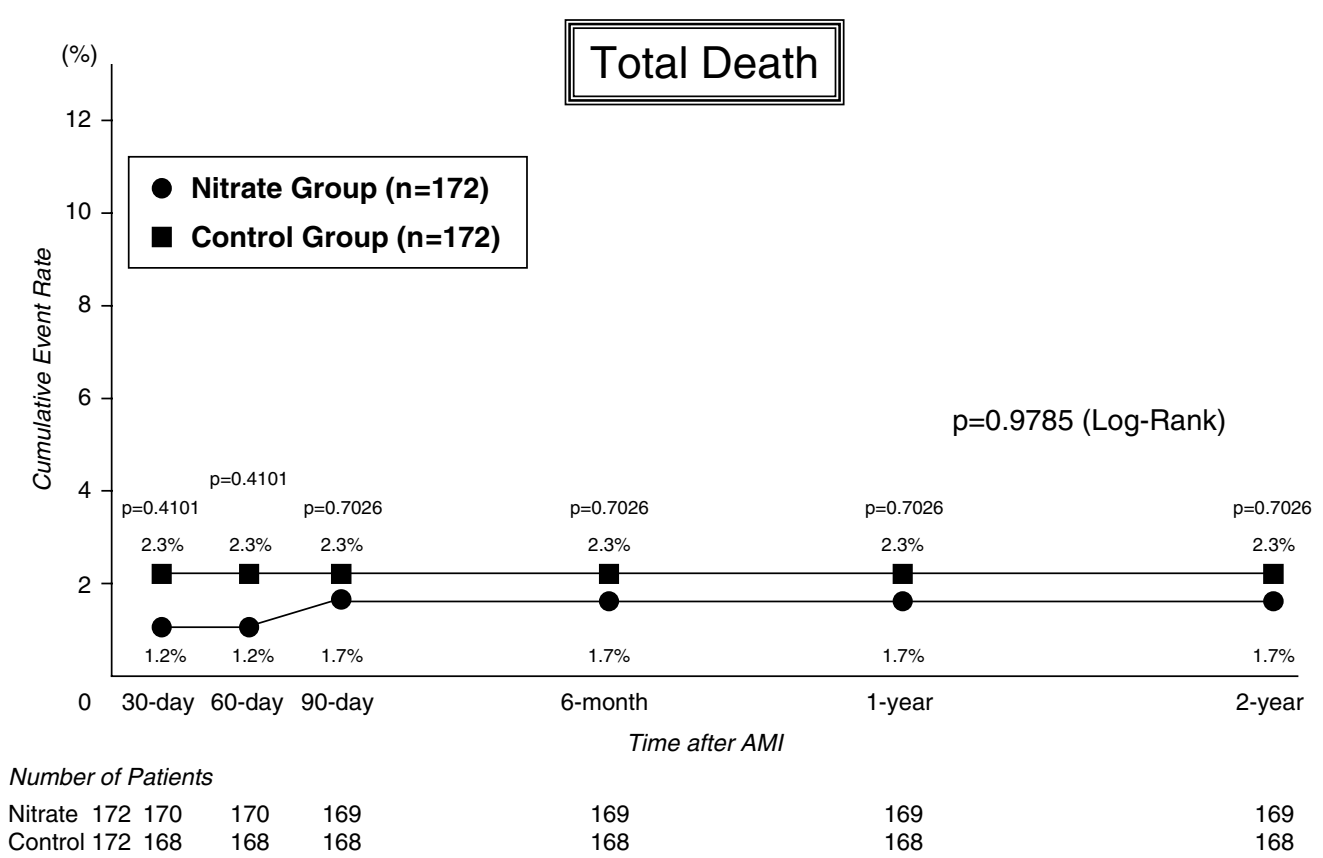

Fig 1. Comparison of cumulative total death rate after propensity score matching in the nitrate group and control group. AMI, acute myocardial infarction.

incidences of diabetes mellitus, hyperlipidemia, preinfarction angina pectoris, ST-segment elevation MI, Q-wave infarction, coronary multivessel involvement, and medications (aspirin, $\beta$-blocker, angiotensin-converting enzyme inhibitor).
Short- and Long-Term Adverse Events

Table 2 shows the all-cause mortality, cardiac events and cardiovascular events in patients treated with and without nitrates. The 30-day, 60-day and 90-day all-cause mortality rates were significantly lower in patients of the nitrate 


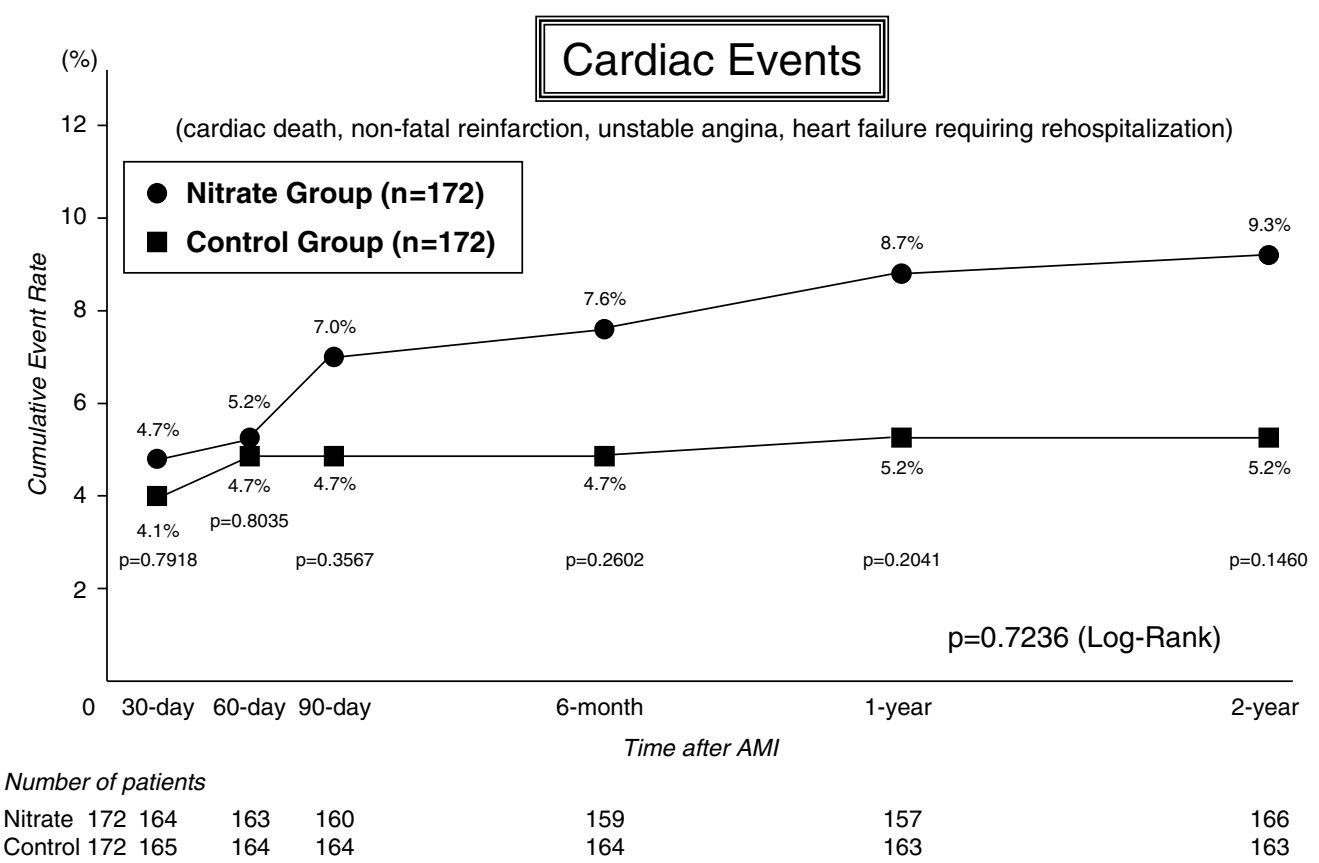

Fig 2. Comparison of cumulative rate of cardiac events (cardiac death, non-fatal reinfarction, unstable angina, heart failure requiring rehospitalization) after propensity score matching between the nitrate group and control group. AMI, acute myocardial infarction.

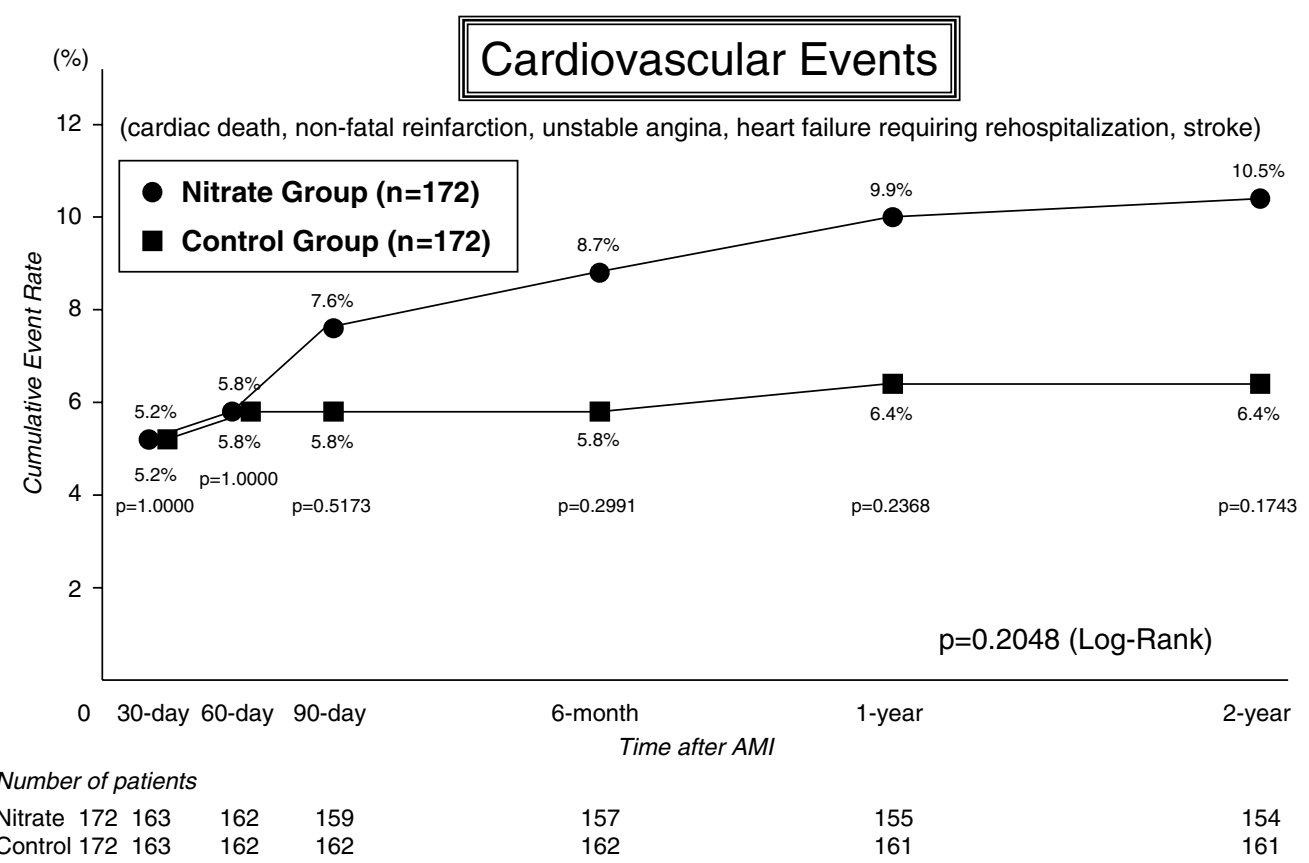

Fig 3. Comparison of cumulative rate of cardiovascular events (cardiac death, non-fatal reinfarction, unstable angina, heart failure requiring rehospitalization, stroke) after propensity score matching between the nitrate group and control group. AMI, acute myocardial infarction.

group than in the non-nitrate control group. However, no significant differences were observed at 6 months, 1 year and 2 years after AMI. Cardiac events at 30 days and 60 days after AMI were more likely in the control group; however, there were no differences between the 2 groups after 90 days. Cardiovascular events were significantly lower only at 30 days after AMI.

\section{Propensity Score Matching}

Propensity score analysis with greedy matching was used to create matched pairs between the nitrate and control groups. In the present study, 172 cases were successfully matched in a 1:1 manner with 172 corresponding controls (Table 3). There were no significant differences between the nitrate and control groups in all-cause mortality (Fig 1), cardiac events (Fig 2) or cardiovascular events (Fig 3) at each 
time point (30 days, 60 days, 90 days, 6 months, 1 year, and 2 years) or during the follow-up periods.

\section{Discussion}

In the present study, we investigated the prognostic significance of long-term nitrate treatment after AMI in the coronary interventional era. Patients treated with nitrates showed good short-term prognosis compared with those who did not take nitrates, without adjusting for patient background. However, nitrate therapy did not improve adverse events after adjusting for all clinical characteristics.

Rapaport et al demonstrated that long-acting nitrate therapy reduced mortality rate during an average of 11 months after $\mathrm{AMI}^{17}$ and the present results showed that nitrates improved 30-day, 60-day and 90-day mortality, 30-day and 60-day cardiac events, 30-day cardiovascular events according to crude comparisons. However, similar to the previous report ${ }^{17}$ the clinical background was markedly different between patients treated with and without nitrates. The prescription of calcium-channel blockers was more frequent in the nitrate group, possibly because of the limited number of patients who underwent PCI or had underlying serious conditions such as higher age, hypertension, high serum creatinine levels, previous MI, coronary multivessel involvement or complication of coronary spasm. Therefore the attending physicians may have considered there was a need for calcium-channel blockers in addition to nitrates. Accordingly, crude comparisons about future events after AMI should not be performed without taking these confounding factors into consideration. In this regard, overall randomized trials indicated that there was risk reduction of mortality with intravenous and oral nitrate treatments?,18 Fitzgerald et al also reported that oral nitrates resulted in non-significant mortality reduction in patients with AMI followed up for 6 months $!^{9}$ These data suggest that nitrate treatment may be beneficial in patients with heart failure caused by moderate or large MI. On the other hand, Ishikawa et $\mathrm{al}^{7}$ and Kanamasa et al ${ }^{8}$ concluded from their prospective studies that long-term treatment with nitrates increased total mortality and cardiac events in patients after AMI during approximately 18-month follow-up period. However, the number of patients and their clinical backgrounds were quite different between patients treated with and without nitrates in those studies, and therefore the data randomization was insufficient.

There is a growing interest in the use of propensity score-based methods for estimating treatment effects in observational studies. The propensity score is defined as a subject's probability of treatment assignment conditional on measured covariates? 20,21 To minimize the effect of selection bias on outcomes, we used the technique of propensity score matching for clinical characteristics and excluded distortion of confounding factors. Based on propensity score matching, nitrate treatment did not have any impact on post-AMI adverse events. The different results reported by previous groups on long-term nitrate treatment are mainly caused by an imbalance of patient background and whether trials were conducted in the prethrombolytic or thrombolytic era. The treatment policy for AMI has also changed over time. Currently in Japan, emergency PCI is aggressively performed in approximately $80 \%$ of patients with AMI and TIMI grade 3 flow of the culprit lesion is achieved in approximately 90\%!10-12 The trend in medical treatment for AMI has also varied widely over the past several decades?22,23
Nitrate tolerance may play an important role in increasing cardiac events. Nitrates can cause free radical production, endothelial dysfunction, and sympathetic activation, which reduce the generation and bioavailability of nitric oxide, and therefore nitrate therapy may have long-term detrimental effects 24 On the other hand, long-term nitrate treatment does not increase vascular superoxide production despite impairment of the relaxant response ${ }^{25}$ The duration of nitrate treatment and follow-up period of the GISSI- $3^{5}$ and ISIS- 46 trials were relatively short and large-scale randomized clinical trials concerning long-term nitrate treatment are required. At this stage, the clinical impact of nitrate tolerance is unclear and the effect of long-term nitrate therapy on clinical outcome is controversial. Importantly, Japanese have diffusely hyperreactive coronary arteries compared with Caucasians ${ }^{26}$ and nitrates have been used for coronary artery disease, including coronary spasm, in many Japanese patients. In the Japanese $\beta$-Blockers and Calcium antagonists Myocardial Infarction (JBCMI) study, the cardiovascular event rate was substantially higher in Western post-AMI patients than in Japanese receiving reperfusion therapy, which may contribute to the lower rates of morbidity and mortality ${ }^{27}$ In this regard, a common form of nitrate withdrawal (rebound) is observed in patients whose angina is intensified after discontinuation of nitrates ${ }^{28-30}$ so abrupt withdrawal after long-term administration of nitrates may be unsafe. A thorough examination is needed of the significance of post-AMI long-term nitrate therapy in the coronary interventional era. In the present study, nitrate therapy had little impact on long-term prognosis but did not result in poor clinical outcome in AMI patients. Therefore, nitrate therapy can be potentially effective in particular cases, such as patients with coronary spasm or heart failure caused by large MI. For secondary prevention, nitrates should be avoided in patients with healed MI who do not have additional myocardial ischemia and heart failure.

\section{Study Limitations}

This study was retrospective in nature. Patients were treated with nitrates soon after hospitalization and continued to receive them, but the amount of long-acting nitrates was not investigated in detail. However, our study included all patients with AMI entered in the 2003 database. All patients were followed after the onset of AMI, and thus the results of the present study should reflect the actual condition of patients with AMI in Japan. Prospective randomized clinical trials are warranted to determine the effects of long-term nitrate treatment for secondary prevention of AMI.

\section{Acknowledgments}

This study was supported by a Research Grant for Cardiovascular Disease (14C-4) from the Ministry of Health, Labour and Welfare.

\section{References}

1. Parker JD, Parker JO. Nitrate therapy for stable angina pectoris. $N$ Engl J Med 1998; 38: 520-531.

2. Morrow DA, Gersh BJ, Braunwald E. Chronic coronary artery disease. In: Braunwald E, editor. Heart disease: A textbook of cardiovascular medicine, 7th edn. Philadelphia: WB Saunders; 2005; $1281-1354$.

3. Yusuf S, Sleight P, Held P, McMahon S. Routine medical management of acute myocardial infarction: Lessons from overviews of recent randomized controlled trials. Circulation 1990; 82(Suppl II): II-117-II-134. 
4. Oral anti-anginal nitrates should be indicated only for single, not chronic, use in absence of long-term data, FDC Cardio-Renal Drugs Advisory CMTE concludes. FDC Reports "The Pink Sheet.” June 7, 1993.

5. Gruppo Italiano per lo studio della sopravvivenza nell'infarto miocardico. GISSI-3: Effects of lisinopril and transdermal glyceryl trinitrate singly and together on 6-week mortality and ventricular function after acute myocardial infarction. Lancet 1994; 343: $1115-$ 1122 .

6. ISIS-4 (Fourth International Study of Infarct Survival) collaborative group. ISIS-4: A randomised factorial trial assessing early oral captopril, oral mononitrate, and intravenous magnesium sulphate in 58050 patients with suspected acute myocardial infarction. Lancet 1995; 345: 669-685.

7. Ishikawa K, Kanamasa K, Ogawa I, Takenaka T, Naito T, Kamata N, et al. Long-term nitrate treatment increases cardiac events in patients with healed myocardial infarction. Jpn Circ J 1996; 60: 779-788.

8. Kanamasa K, Hayashi T, Takenaka T, Kimura A, Ikeda A, Ishikawa K. Chronic use of continuous dosing of long-term nitrates does not prevent cardiac events in patients with severe acute myocardial infarction. Cardiology 2000; 94: 139-145.

9. Nagao K, Kanmatsuse K, Ooiwa K, Satou K, Watanabe I, Yamasita $\mathrm{M}$, et al. The effects of long-acting nitrates on 5-year cardiac events of patients with coronary thrombolytic therapy for acute myocardial infarction. Intern Med 2000; 39: 877-884.

10. Kojima S, Sakamoto T, Ishihara M, Kimura K, Miyazaki S, Yamagishi M, et al. Prognostic usefulness of serum uric acid after acute myocardial infarction (The Japanese Acute Coronary Syndrome Study). Am J Cardiol 2005; 96: 489-495.

11. Ishihara M, Kojima S, Sakamoto T, Asada Y, Kimura K, Miyazaki $\mathrm{S}$, et al. Usefulness of combined white blood cell count and plasma glucose for predicting in-hospital outcomes after acute myocardial infarction. Am J Cardiol 2006; 97: 1558-1563.

12. Kosuge M, Kimura K, Kojima S, Sakamoto T, Ishihara M, Asada Y, et al. Sex differences in early mortality of patients undergoing primary stenting for acute myocardial infarction. Circ J 2006; 70: 217 221.

13. WHO Study Group. Prevention of diabetes mellitus: Report. Tech Rep Ser No. 844. Geneva: World Health Organization, 1994.

14. TIMI Study Group. The Thrombolysis in Myocardial Infarction (TIMI) trial: Phase I findings. N Engl J Med 1985; 312: 932-936.

15. Killip T 3rd, Kimball JT. Treatment of myocardial infarction in a coronary care unit: A two year experience with 250 patients. Am J Cardiol 1967; 20: 457-464.

16. Parsons LS. Reducing bias in a propensity score matched-pair sample using greedy matching techniques. In: Proceedings of the Twentysixth Annual SAS Users Group International Conference 2001. SAS Institute Inc, Cary (NC); April; 22-25.

17. Rapaport E. Influence of long-acting nitrate therapy on the risk of reinfarction, sudden death, and total mortality in survivors of acute myocardial infarction. Am Heart J 1985; 110: 276-280.

18. Held P, Teo KK, Yusuf S. Effects of beta-blockers, calcium channel blockers and nitrates in acute myocardial infarction and unstable angina pectoris. In: Topol EJ, editor. Interventional cardiology. Philadelphia, WB Saunders; 1990; 49-65.

19. Fitzgerald LJ, Bennett ED. The effects of oral isosorbide 5-mononitrate on mortality following acute myocardial infarction: A multicenter study. Eur Heart J 1990; 11: 120-126.

20. Rubin DB. Estimating causal effects from large data sets using propensity scores. Ann Intern Med 1997; 127: 757-763.

21. D'Agostino RB Jr. Propensity score methods for bias reduction in the comparison of a treatment to a non-randomized control group. Stat Med 1998; 17: 2265-2281.
22. Ishikawa K, Kimura A, Taniwa T, Takenaka T, Hayashi T, Kanamasa $\mathrm{K}$, et al. Modification of treatment strategies over a period of 14 years has markedly reduced cardiac events among post-myocardial infarction patients. Circ J 2002; 66: 881-885.

23. Kusuoka H, Koretsune Y, Chino M, Nishiyama K, Shiraki T, Nakamura K, et al. Disparity in the process and outcome of the treatment for acute myocardial infarction in Japan: CAMPAIGN study in the National Hospital Network. Circ J 2005; 69: 1447-1453.

24. Gori T, Parker JD. Nitrate tolerance: A unifying hypothesis. Circulation 2002; 106: 2510-2513.

25. Muller S, Laber U, Mullenheim J, Meyer W, Kojda G. Preserved endothelial function after long-term eccentric isosorbeide mononitrate despite moderate nitrate tolerance. J Am Coll Cardiol 2003; 41: 1994-2000.

26. Beltrame JF, Sasayama S, Maseri A. Racial heterogeneity in coronary artery vasomotor reactivity: Differences between Japanese and Caucasian patients. J Am Coll Cardiol 1999; 33: 1442-1452.

27. The Japanese, Beta-blockers and Calcium Antagonists Myocardial Infarction (JBCMI) Investigators. Comparison of the effects of beta blockers and calcium antagonists on cardiovascular events after acute myocardial infarction in Japanese subjects. Am J Cardiol 2004; 93: $969-973$.

28. Przybojewski JZ, Heyns MH. Acute coronary vasospasm secondary to industrial nitroglycerin withdrawal: A case presentation and review. S Afr Med J 1983; 63: 158-165.

29. Waters DD, Juneau M, Gossard D, Choquette G, Brien M. Limited usefulness of intermittent nitroglycerin patches in stable angina. $J$ Am Coll Cardiol 1989; 13: 421-425.

30. Ferratini M. Risk of rebound phenomenon during nitrate withdrawal. Int J Cardiol 1994; 45: 89-96.

\section{Appendix 1}

JACSS Principle Investigators

Ogawa H (Chairperson; Kumamoto University), Asada Y (Miyazaki Medical College), Tei C (Kagoshima University), Kimura K (Yokohama City University Medical Center), Tsuchihashi K (Sapporo Medical University), Ishihara M (Hiroshima City Hospital), Miyazaki S, Yamagishi M, Ikeda Y (National Cardiovascular Center), Shirai M (Yamaguchi University), Hiraoka H (Osaka University), Shimoyama N (Oita National Hospital), and Sonoda M (National Hospital Kyushu Cardiovascular Center).

JACSS Participating Institutions and Clinical Investigators

Ogata Y (Japanese Red Cross Kumamoto Hospital), Honda T, Sakamoto $\mathrm{T}$ (Social Welfare Organization Imperial Gift Foundation Incorporated Saiseikai Kumamoto Hospital), Hokamura Y (Kumamoto City Hospital), Saito T (Kumamoto Central Hospital), Mizuno Y (Kumamoto Kinoh Hospital), Miyagi H (Kumamoto National Hospital), Matsumura T (Labor Welfare Corporation Kumamoto Rosai Hospital), Tabuchi T (Yatsushiro Health Insurance General Hospital), Sakaino N (Amakusa Medical Center), Kimura K (Arao City Hospital), Obata K (Health Insurance Hitoyoshi General Hospital), Shimomura H (Fukuoka Tokushukai Medical Center), Matsuyama K (Social Insurance Ohmuta-Tenryoh Hospital), Nakamura N (Shinbeppu Hospital), Yamamoto N (Miyazaki Prefectural Nobeoka Hospital), Hase M (Sapporo Medical University School of Medicine), Matsuki T (Shinnittetsu Muroran General Hospital), Hashimoto A (Kushiro City General Hospital), Abiru M (Oji General Hospital), Matsuoka T (National Hospital Kyusyu Cardiovascular Center), Toda H, Ri S (Kagoshima City Hospital), Toyama Y, Yamaguchi H, Toyoshima S (Nanpuh Hospital), Torii H (Kagoshima Medical Association Hospital), Atuchi Y, Miyamura A (Tenyokai Chuo Hospital), Hamasaki S (Kagoshima University Faculty of Medicine), Miyahara K (Shinkyo Hospital) and Kojima S, Matsui K (Kumamoto University Hospital). 